\title{
Active Knowledge Models and Enterprise Knowledge Management
}

\author{
Frank Lillehagen ${ }^{1}$ and John Krogstie ${ }^{2}$ \\ ${ }^{\prime}$ Computas AS, Norway, ${ }^{2}$ SINTEF, Norway, fli@computas.com
}

\begin{abstract}
We present in this paper a novel approach for integrating enterprise modelling and knowledge management in dynamic networked organisations. The approach is based on the notion of active knowledge models $\left(\mathrm{AKM}^{\mathrm{TM}}\right)$. An AKM is a visual model of enterprise aspects that can be viewed, traversed, analysed, simulated, adapted and executed by industrial users.

To integrate particular process technologies from the enterprise perspective of generic business process types to the individual work tasks at the instance level, our work is based on our process modelling reference model. It identifies 4 layers of process knowledge representation, from general process logic to actual, situated work performance. Process modelling occurs at several levels concurrently, and may start at any level. Learning within and between levels is supported using a framework for process knowledge management.
\end{abstract}

\section{INTRODUCTION}

The business environment is getting increasingly dynamic. Co-operation across traditional organizational boundaries is increasing, as outsourcing and electronic business is enabled by the Internet and IS in general. When such co-operation moves beyond the buying and selling of goods and welldefined services, there is a need for a flexible infrastructure that supports not only information exchange, but also knowledge sharing, creation, utilisation and management within and across the traditional organizational borders. To address these challenges, new organizational forms, such as different types of virtual Organizations and extended enterprises flourish. This demands a 
new approach to enterprise integration and system engineering. Our approach to this area is the use of Active Knowledge Models (AKM).

An Active Knowledge Model is a visual externalisation of knowledge of enterprise aspects that can be operated on (viewed, traversed, analysed, simulated, adapted and executed) by industrial users. What does it mean that the model is active? First of all, the visual model must be available to the users of the information system at runtime. Second, the model must influence the behaviour of the computerised support system. Third, the model must be dynamic, users must be supported in changing the model to fit their local situation, enabling tailoring of the system's behaviour.

\section{THE AKM APPROACH}

AKMs of Enterprises imply that the enterprise is extended by distributed team working on layers of knowledge, and that simultaneous modelling, meta-modelling and work can be performed.

AKM implementation is dependent on a rich generic knowledge base and powerful development and extension capabilities of the infrastructure. Being able to support collaborative work and managing knowledge will decide the quality of the solution, of the methodology, and of the knowledge and solutions created. The usage and value of the solution is mainly decided by the infrastructure, but also by the competence and knowledge of the teams involved.

\subsection{Enterprise modelling and knowledge management}

The concept of knowledge management has been used in different disciplines, previously mostly in knowledge management and engineering (Skyrme, Amidon, 1997, Schreiber, et al. 2000). Knowledge management is mainly understood by practitioners from manufacturing and the service industry as part of corporate culture and a business-oriented method as "The sum of procedures to generate, store, distribute and apply knowledge to achieve organisational goals".

All main approaches to knowledge management emphasise the process character with inter-linked tasks or activities. Business process modelling is usually done for very specific goals, which partly explains the great diversity of approaches found in literature (Vernadat, 1996) and practice. The main reasons for doing BPM are:

a) To improve human understanding and communication

b) To guide system development 
c) To provide computer-assisted analysis through simulation or deduction

d) To enable model deployment and activation for decision making and operation monitoring and control

There are four major knowledge dimensions in any enterprise:

- Products and Services, the results of work and the deliverables of projects

- Organization and People, competence and skills, and resources for work performance.

- Processes and Tasks, including work breakdown structures for different purposes.

- Systems and Tools, technical infrastructure with architectures, interfaces and tools.

The AKM, irrespective of purpose and scope, will always take one or more views from all the four main dimensions into consideration. Which aspects and views to model also depend on the audience and the intended use of the model? The AKM approach is also a holistic approach leaving it to the developers and the users to decide which views, aspects of structures and flows, and which operational solutions should constitute the model to meet expectations and satisfy users and audience.

To integrate in particular process technologies from the enterprise perspective of generic business process types to the individual work tasks at the instance level, our work is based on extending our process modelling reference model (Jørgensen, Carlsen, 1999) shown in Fig. 1. It identifies 4 layers of process knowledge representation, from general process logic to actual, situated work performance. Process modelling occurs at several levels concurrently, and may start at any level.

Layer 1 - Describe Process Logic: At this layer, we identify the constituent activities of generic, repetitive processes and the logical dependencies between these activities. A process model at this layer should be transferable across time and space to a mixture of execution environments. Examples of process logic are conceptual value chains and best practice-models of "ways of working" for particular types of organisations.

Layer 2 - Engineer Activities: Here process models are expanded and elaborated to facilitate business solutions. Elaboration includes concretisation, decomposition, and specialisation. Integration with local execution environment is achieved e.g. by describing resources required for actual performance.

Layer 3 - Manage Work: The more abstract layers of process logic and of activity description provide constraints but also useful resources (in the form of process templates) to the planning and performance of each extended enterprise process. At layer 3, more detailed decisions are taken re- 
garding the performance of work in the actual work environment with its organizational, information, and tool resources; the scope is narrowed down to an actual process instance. Concrete resources increasingly are intertwined in the model, leading to the introduction of more dependencies. Management of activities may be said to consist of detailed planning, coordination and preparation for resource allocation.

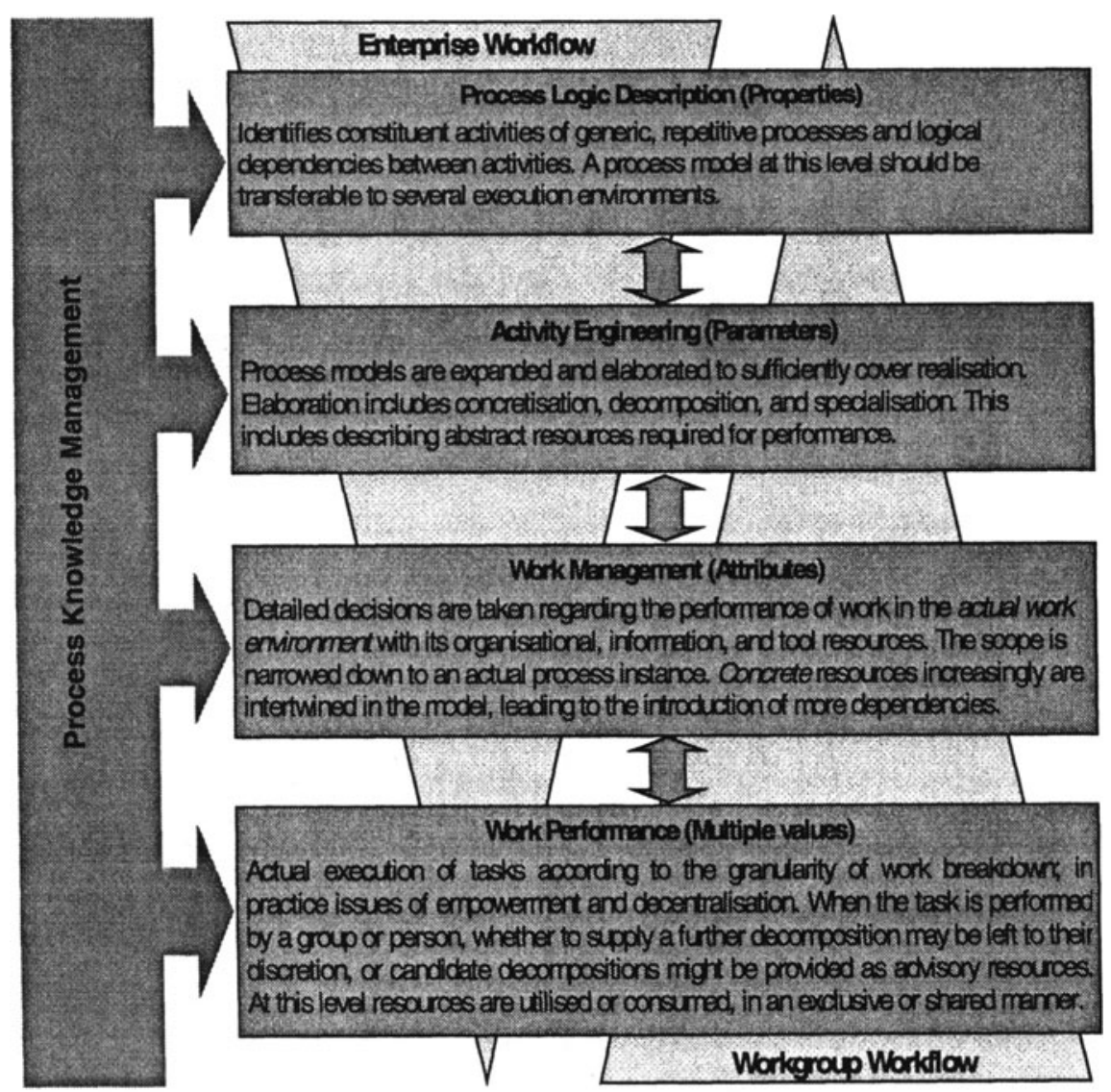

Figure 1: Process modelling reference model

Layer 4 - Perform Work: This lowest layer of the model covers the actual execution of tasks according to the determined granularity of work breakdown, which in practice is coupled to issues of empowerment and decentralisation. When a group or person performs the task, whether to supply a further decomposition may be left to their discretion, or alternative candidate decompositions might be provided as advisory resources. At this layer resources are utilised or consumed, in an exclusive or shared manner. 
Process knowledge management can be defined as the collection of processes necessary for innovation, dissemination, and exploitation of knowledge in a co-operating ensemble where knowledge seekers are linked to knowledge sources and a shared knowledge base is cultivated. Process knowledge management is active at all layers of the model, which will be described in more detail below based on (Jørgensen, 2000). Here, our main concern is to understand the mechanisms that enable us to integrate process models at various levels of abstraction, so we need a framework that show the activities involved in converting between general (layer 1 and 2) and particular (layer 3 and 4) models. Fig. 2 shows the reference model we have chosen.

Applying a general process model to a particular situation is a case of reuse (Reuse may also refer to copy and paste of a previously developed particular model into a new process, i.e. reuse must not always occur via a general model. Copy and paste reuse is important to minimise the effort of model building, but less useful for organizational process improvement and knowledge management). Reuse involves selecting a process type (general model) and using it to generate a particular model for process enactment.

In some enterprise modelling and process improvement initiatives, particular models are seldom used. For such initiatives to be cost effective, they must

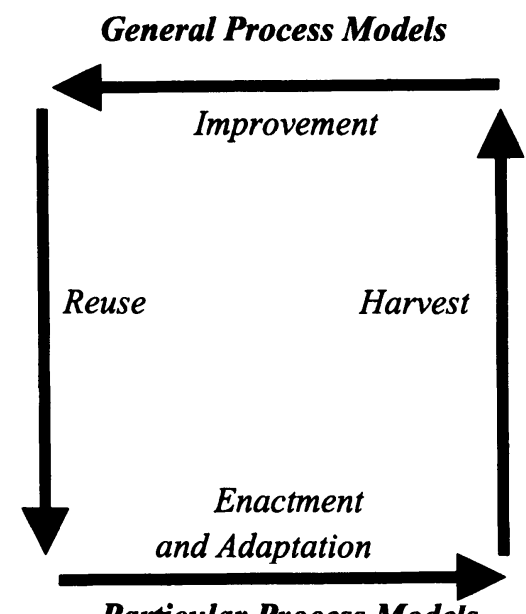

Particular Process Models

Figure 2: Lifecycle of process model evolution target general models that are used in several actual processes. The process of transforming one or more particular models into a general one is called harvesting. The goal of harvesting is to provide and update templates that may be reused in the future, and to utilise practical experience as an input to assessment and improvement of the general models. Templates include personal, group, and organizational fragments, process examples and patterns, in addition to complete definitions of routine procedures. Following traditional terminology within software process modelling, the activity where people assess and update general models is called process improvement. The use and dynamic adaptation of particular models during performance of work, is called process enactment.

The activities of process enactment, harvesting, improvement and reuse form a complete learning cycle. If one activity is not performed, the others will not be as effective. This does not imply that all activities need to be ex- 
plicit or encoded in software. A user may for instance improve a template based on lessons learned in a project, even without software support for harvesting from the particular project model. Similarly, a project model may act as a passive plan and influence practice although automated enactment support is not available.

\section{EXTERNAL INFRASTRUCTURE AND APPROACH}

In the EXTERNAL project IST 1999-10091 (EXTERNAL, 2000) we are working further to develop a technical and conceptual infrastructure to support the AKM approach as a basis for enterprise knowledge management through process knowledge management.

The most innovative contributions from the EXTERNAL can be summarised as:

- Implementing an Extended Enterprise (EE) based on new capabilities from AKM technology, exploiting meta-models as enterprise integrators and technology convergence enablers.

- Implementing the multiple views of active objects, exploiting the reflective, recursive, repetitive and replicable nature of (situated) work process knowledge. Software methods are defined and linked as properties of visually engineered and managed objects.

- Applying the model evolution and management processes that are enabled by parallel and commercially developed solutions based on the same core concepts and common meta-models.

- Implementing a four-layered infrastructure with open enterprise formation and operation capabilities and architectures for dynamic IT component inclusion, knowledge representation, work and model management and dynamic user environment generation.

- Implementing an integrated methodology supported by the layered infrastructure.

The infrastructure, methodology, case-study solutions, and the EXTERNAL project itself are developed in parallel. The layered infrastructure (Lillehagen, 2002a) will support and implement the methodology, provide project management services, and implement work process driven solutions from re-composable knowledge and software.

Version 1.0 of the infrastructure is an integration of the enterprise and process modelling applications brought into the EXTERNAL project by the partners and further extended there. The following tools provide the core software services of the technical layer.

- METIS, a general purpose enterprise modelling and visualisation tool, 
- XChips, a co-operative hypermedia tool integrated with process support and synchronous collaboration,

- SimVision, a project simulator used to analyse resource allocation, highlighting potentials for delays and backlogs.

- Workware, an emergent workflow management system with to-dolists, document sharing, process enactment and awareness mechanisms.

Together these tools offer varied functionality for creating, maintaining, and utilising shared active knowledge models of the extended enterprise. The models are managed through a shared repository residing on a web server. For the representation and interchange of models, an XML DTD is defined.

As mentioned above, the infrastructure is best described as consisting of four layers. These layers are identified as:

Layer 1, the ICT layer: - defining and describing the ICT platform, the software architectures, tools, software components and capabilities, connectivity and communications. The ICT layer supports multi-user access control and repository management. The architecture has 3-tiers, clients, application servers, and data servers (web services), i.e. server applications communicating with its clients solely through standard web protocols such as HTTP and exchanging data in XML over SOAP.

Layer 2, the Knowledge Representation layer: - defining and describing constructs for knowledge model representation, developing, sharing and managing the contents of model and meta-model repositories. The Knowledge Representation layer defines how models, meta-models and meta-data are represented, used and managed. METIS is used to manage models, modelling languages and meta-data. The model content can be persistently stored in the shared model repository. Future versions will support project, team and work administrative processes and an administrative database. Model contents, meta-model versions, revisions and variants, and meta-data hierarchies that are local, project specific or global will be separately managed. The architecture involves work processes that manage the project administration database (organisation, roles, users) and the meta-model repository, and that save accumulated experiences and life histories for change and configuration management and situated learning purposes.

Layer 3, the Model and Work Management layer; - modelling the customer solution, adapting engineering processes, and implementing work processes, executing and managing models. Model and Work Management will model and implement work processes for the engineering processes, and provides services to support the EE teams. In versions 1.5 and 2.0 we will model and implement work processes as active, reflective objects. Model and work management will therefore be implemented as immersed, rule driven and reflective work processes. The architecture of this layer is the 
management rules embedded in use case work processes, the model engineering work processes, and the life-cycle management model automatically creating life-history, when teams are executing work processes.

Layer 4, the Work Performance layer; - implementing customer solutions, generating work environments as personalised and context-sensitive views and GUI's being worktops accessed through portal-based user environments, and performing work with life-cycle management control.

\section{RELATED WORK}

With respect to supporting dynamically networked organisations, most B2B E-business frameworks (Shim, 2000) focus on information exchange and business transactions. This is also the case with newer frameworks such as ebXML and the perceived uses of Web Services. These approaches lack support for the dynamic, collaborative, and knowledge-intensive parts of inter-organisational processes, and knowledge management in this setting.

The major application area of BPM is still Business-Process Reengineering (BPR) and Business-Process Optimisation. The real potential of BPM real time decision support - is barely exploited.

Enterprise ontologies have been proposed as a way of solving the communication problems arising from different interpretative frameworks in different organisations. This approach is based on conventional notions of model interpretation, i.e. the Turing paradigm, where the technical actor interpretation is fully automated and no interaction is allowed to aid interpretation, and not the more powerful interaction machine paradigm (Jørgensen, 2001; Wegner, 1999). The main characteristic of an interaction machine is that it can pose questions to human actors (users) during its computation. The problem solving process is no longer just a user providing input to the machine, which then processes the request and provides an answer (output); it is a multi-step conversation between the user and the machine, each being able to take the initiative.

Workflow management systems have also been proposed as a solution for inter-organisational collaboration (van der Aalst, Weske, 2001). Knowledge intensive processes are found to require a degree of flexibility not enabled by conventional production workflow systems. Alternative such as Service-flow (Wetzel, 2002) is appearing, but these new approaches are not linked to explicit process modelling. 


\section{CONCLUSION AND FURTHER WORK}

The next version of the infrastructure will be released towards in the beginning of 2002, and we are currently collecting experiences from the case studies as input to further developments. First experiences are reported in (Lillehagen, 2002b), where parts of a quasi-experimental investigation are reported. This paper is focusing specifically on the results reported on communication, learning and trust in an extended enterprise being supported by our model-based infrastructure. Positive trends have been identified within all these areas, making us convinced of the great potential of active knowledge models in this area. Version 2.0 of the 4-layer infrastructure is planned to be available in September 2002. Focus is on implementing EE capabilities as repeatable and reusable work processes and services at layers 2, 3 and 4 of the infrastructure.

\section{REFERENCES}

Aalst, W. v. d., Desel, J. Oberweis, A. (2000), Business Process Management. LNCS 1806, Springer-Verlag.

EXTERNAL (2000-2002), EXTERNAL - Extended Enterprise Resources, Networks And Learning, EU Project, IST-1999-10091.

Jørgensen, H. D. Carlsen, S. (1999), Emergent Workflow: Integrated Planning and Performance of Process Instances, Workflow Management '99, Münster, Germany.

Jørgensen, H. D. (2000), Software Process Model Reuse and Learning, in Proceedings of Process Support for Distributed Team-based Software Development (PDTSD'00), Orlando, Florida. IIIS - International Institute of Informatics and Systemics.

Lillehagen, F. Dehli, E. Fjeld, L. Krogstie, J. Jørgensen, H. D. (2002a), Utilizing active knowledge models in an infrastructure for virtual enterprises, Proc. PROVE'02 IFIP Conference on infrastructures for virtual enterprises, Portugal, May, Kluwer.

Lillehagen, F., Krogstie, J., Jørgensen, H. D., Hildrum, J. (2002b), Active Knowledge Models for supporting eWork and eBusiness. Accepted at ICE'2002, Rome, June.

Schreiber A. Th., Hoog, R., Akkermans, H., Anjewierden, A., Shadbolt, N., Velde W. (2000), Knowledge Engineering and Management. The CommonKADS Methodology. The MIT Press, Cambridge, London.

Shim, S. S. Y., Pendyala, V. S., Sundaram, M. and Gao, J. Z. (2000), Business-to-Business ECommerce Frameworks, IEEE Computer, vol. 33, no. 10.

Skyrme, D.J., Amidon, D.M. (1997), Creating the Knowledge-Based Business. Business Intelligence, London, New York.

Vernadat, F. (1996) Enterprise Modelling and Integration. Chapman and Hall.

Wegner, P. Goldin, D. (1999), Interaction as a Framework for Modeling, in Conceptual Modeling. Current Issues and Future Directions, Lecture Notes in Computer Science 1565, P. P. Chen, J. Akoka, H. Kangassalo, and B. Thalheim, (Eds.), Springer-Verlag.

Wetzel, I. Klischewski, R. (2002), Serviceflow beyond Workflow? Concepts and Architectures for Supporting Interorganizational Service Processes. In Pidduck, A. B., Mylopoulos, J. Woo, C. C. and Ozsu, M. T. (Eds.), Proceeding s from CaiSE'14, Toronto, Canada. 